\section{Schlafstörungen}

\begin{tabular}{|l|c|c}
$47-$ Warum liegt dieser Patient wach? \\
50 - Gestörte Nachtruhe bei \\
Senioren \\
Prof. Dr. med. Adrian Gillissen \\
Klinik für Lungen- und Bronchialmedizin, Klinikum Kassel \\
Schlafen schlecht
\end{tabular}

„... die ganze Nacht kein Auge zugetan!“

\title{
Zehn Tipps zum Einschlafen
}

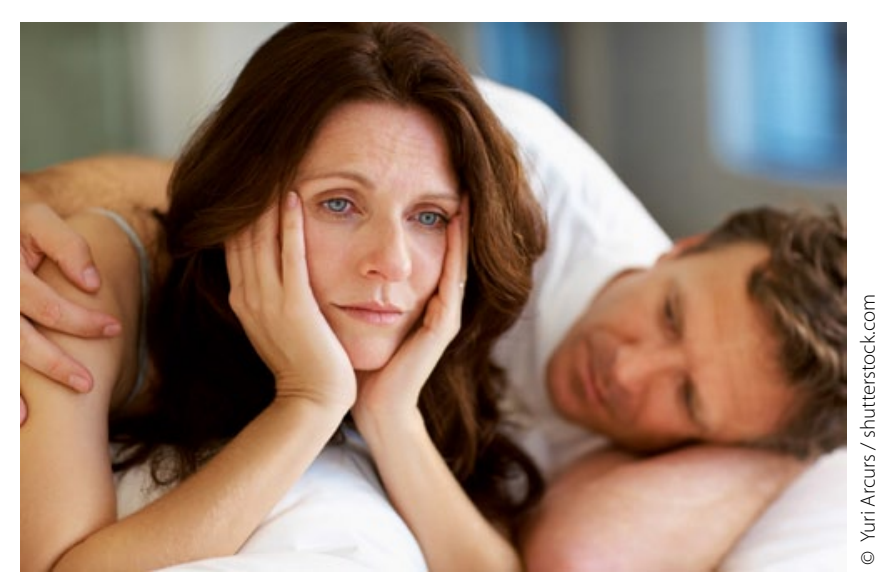

- Ein nicht erholsamer Schlaf beeinflusst wesentlich die Lebensqualität, Schlafentzug wurde schon als Foltermethode eingesetzt, und viele Erkrankungen sind direkt oder indirekt mit Schlafstörungen assoziiert.

Insomnien sind durch Störungen beim Einschlafen, häufiges Erwachen in der Nacht, sehr frühes Erwachen am Morgen und / oder schlechte Schlafqualität definiert. Fast jeder 6. Erwachsene leidet heute unter einer mehr oder weniger ausgeprägten Schlafstörung. Weitere 13-15\% leiden unter gelegentlichen und leichten Schlafstörungen. Ältere Menschen sind häufiger betroffen als jüngere. Bei den über 60-Jährigen leidet etwa jeder 4. regelmäßig an Insomnie. Die Häufigkeit obstruktiver Schlafapnoesyndrome wird mit ca. 5\% angegeben, Restless-Legs-Syndrome liegen bei $4 \%$ vor. Sogar die Narkolepsie wird noch mit 0,5\% angegeben.

Als Arzt ist man nahezu zwangsläufig mit Schlafproblemen und ihren Ursachen und Folgen konfrontiert. In diesem Heft werden Schlafstörungen von der neurologischen, psychiatrischen und internistisch-pneumologischen Seite her beleuchtet. Das Patientenmanagement beginnt mit Tipps zum Schlafverhalten (Schlafhygie- ne), setzt sich fort über eine differenzierte Diagnostik und endet mit zielgerichteten Therapieempfehlungen.

Folgende Tipps sollen helfen, zu einem gesunden und erholsamen Schlaf zu finden:

- Haben Sie Schwierigkeiten, nachts zu schlafen, so verzichten Sie auf ein Nickerchen während des Tages.

- Betreiben Sie regelmäßig Sport oder trainieren Sie. Körperliche Bewegung bewirkt normalerweise, dass der Körper sich zufrieden, aber auch müde anfühlt. Achten Sie jedoch darauf, Ihr Training spätestens drei Stunden vor dem Schlafgehen abgeschlossen zu haben. Während des Trainings steigt die Körpertemperatur an und benötigt danach etliche Stunden, um auf die ursprüngliche Körpertemperatur abzukühlen. Eine niedere Körpertemperatur wiederum bewirkt, dass man leichter einschläft und durchschläft.

- Vermeiden Sie Alkohol, Nikotin und Koffein ab ca. sechs Stunden vor dem Schlafengehen. Diese Substanzen verbleiben zwischen drei und fünf Stunden im Körper und können Einschlafphase, Intensität und Erholungswirksamkeit des Schlafes beeinträchtigen.

- Trinken Sie vor dem Zubettgehen wenig und vermeiden Sie schwere oder zu scharfe Gerichte (Sodbrennen!) als Abendmahlzeit. - Gehen Sie nicht hungrig zu Bett! Kohlenhydrathaltige Snacks können eine positive Auswirkung auf die Schlafgewohnheit haben. - Nehmen Sie vor dem Schlafengehen ein entspannendes Schaumbad, hören Sie Entspannungsmusik oder lesen Sie ein paar Seiten eines leichten Romans.

- Trinken Sie vor dem Zubettgehen eine Tasse Johanniskrauttee mit einem Löffel Honig.

- Achten Sie darauf, morgens immer zur gleichen Zeit aufzustehen (wenn möglich, auch am Wochenende). Dieser Schlaf-Wach-Rhythmus trägt wesentlich zu einem gesunden, problemlosen Schlaf bei.

- Haben Sie trotzdem Schwierigkeiten einzuschlafen, stehen Sie auf und hören Sie sich eine CD mit Entspannungsmusik an.

- Setzten Sie sich nicht unter Druck! Je mehr inneren Druck Sie auf sich ausüben, um so schwieriger wird das Einschlafen sein. 